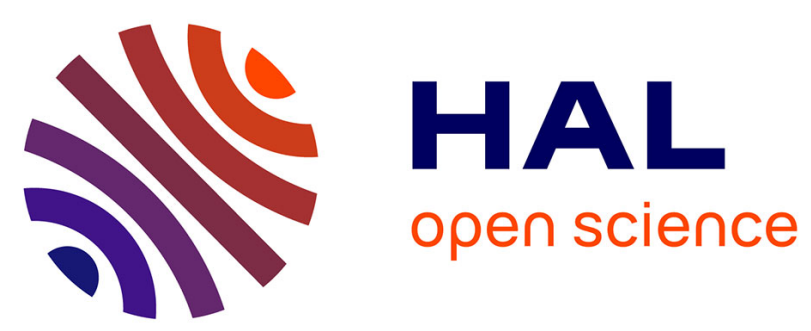

\title{
Anticipatory and Compensatory e-Assistance for Haptic Shared Control of the Steering Wheel
}

Béatrice Pano, Philippe Chevrel, Fabien Claveau

\section{To cite this version:}

Béatrice Pano, Philippe Chevrel, Fabien Claveau. Anticipatory and Compensatory e-Assistance for Haptic Shared Control of the Steering Wheel. 2019 18th European Control Conference (ECC), Jun 2019, Naples, France. pp.724-731, 10.23919/ECC.2019.8796050 . hal-02378864

\section{HAL Id: hal-02378864 \\ https://hal.science/hal-02378864}

Submitted on 25 Nov 2019

HAL is a multi-disciplinary open access archive for the deposit and dissemination of scientific research documents, whether they are published or not. The documents may come from teaching and research institutions in France or abroad, or from public or private research centers.
L'archive ouverte pluridisciplinaire HAL, est destinée au dépôt et à la diffusion de documents scientifiques de niveau recherche, publiés ou non, émanant des établissements d'enseignement et de recherche français ou étrangers, des laboratoires publics ou privés. 


\title{
Anticipatory and Compensatory e-Assistance for Haptic Shared Control of the Steering Wheel
}

\author{
Béatrice Pano ${ }^{1}$, Philippe Chevrel ${ }^{1}$ and Fabien Claveau ${ }^{1}$
}

\begin{abstract}
Shared control is a solution which already showed beneficial effects during experiments for steering control in the automotive sector. It allows improving the lane following performance of the driver by supporting them without losing the benefits of having a human in the loop, as a fallback solution in unexpected situations. This article presents a novel shared control strategy for car steering, using an original two parts method. On the one hand, the feedforward part consists of a trajectory generator based on the simulation of a virtual autonomous vehicle. On the other hand, a mixed $H_{2} / H_{\infty}$ control law constitute the feedback part applied on the difference between the system's state and the virtual system's state. A first benefit of such an architecture is that the two parts can be designed sequentially. Secondly, the virtual vehicle used can lead to any kind of shared control assistance; lane keeping system, but also more generic trajectories such as obstacle avoidance, lane changes, etc... Finally, the sharing level between the human driver and the assistance is made explicit and could be modified on the fly. This strategy was tested by simulating the drivervehicle-road system. Results obtained are satisfying for both lane following and steering sharing between the e-copilot and the driver.
\end{abstract}

\section{INTRODUCTION}

Automating driving tasks has been one of the major research challenge in the last decades. Many issues remain unsolved and safe driving is not guaranteed in every situation. That's why alternative solutions are investigated, as shared control applied on car steering. According to Abbink [1], "In shared control, human(s) and robot(s) are interacting congruently in a perception-action cycle to perform a dynamic task, that either the human or the robot could execute individually under ideal circumstances". This method allows to assist the driver for steering task without losing the benefits to have the human in the loop. It was already shown that it improves lane following performance of the system [2], [3], [4] at the same time as reducing the driver workload [4]. Haptic shared control also allows to reduce some problems that can be met with fully automated car as loss of skill and of situation awareness [5].

Haptic shared control involves a haptic interface: in the steering control case, this interface is the steering wheel. Both the human and the automation can act on the steering wheel and have the knowledge of each other's actions through this interface. This communication is crucial to have an efficient shared control [1]. Moreover, it's important that the driver understands automation's actions for driver comfort and to avoid confusion [5]. Using a human-centered automation system can improve the understanding between driver and automation [5], that's why shared control systems are often based on a driver model [2], [3], [6], [7].

The shared control strategy developed in this article uses the driver model described in [8], [9]. It's a representative cybernetic model which includes a perception and a neuromuscular model of the driver. This model was already used in previous shared control strategy and has resulted in improvements of sharing performance with the driver and in an increased driver comfort [3], [10].

The control method developed here relies on a two parts architecture described as follows: a first anticipatory part which consists of a reference trajectory generator and a compensatory part. These two parts are intrinsically linked, as the feedback terms are based on the feedforward ones (see Section III.A for a short description). This separation is commonly used for autonomous vehicle [11]-[13]. These kind of systems generally use an absolute coordinate system and a steering angle as a command signal to steer the vehicle. In the current study the vehicle position is known relatively to the road lane, and the command signal is a torque applied on the steering wheel. The torque command allows to employ this strategy in an haptic shared control context [1]. Indeed, in this case, it's important to assess and control the effort applied by the assistance which can't be done with the steering angle. The $\mathrm{H}_{2}$-preview solution submitted in [14] also meet these characteristics. However, it implies to know the drivervehicle-road (DVR) system state including the driver model state variables which are not measurable. The shared control proposed is much more flexible. First, it relies on output (rather than state) feedback, therefore it doesn't require the use of an observer to estimate e.g. the driver model state. Moreover, it helps to take into account the robustness, having the mean to use multi-model and multi-objective design. Finally, it makes the control system easily compatible with a possible reference trajectory proposed at the tactical level of autonomous vehicles.

The paper is organized as follows; Section II presents the vehicle-road and driver models used to design the lateral assistance. In Section III the general architecture of the shared control proposed is introduced and discussed. Section IV develops the design methodologies for both anticipatory assistant (using trajectory generation), and compensatory assistant (using feedback on the vehicle-road model). The first one is based on a virtual vehicle model driven by a $H_{2}$-preview controller, and the second one on a static output feedback
${ }^{1}$ IMT-Atlantique, LS2N UMR CNRS (Laboratoire des Sciences du Numérique de Nantes), 44307 Nantes, France (e-mails: firstname.lastname@imt-atlantique.fr). 
TABLE I. DRIVER MODEL PARAMETERS

\begin{tabular}{|c|c|c|}
\hline$K_{p}$ & Anticipation Gain & 3.4 \\
\hline$K_{c}$ & Compensation Gain & 15 \\
\hline$T_{I}$ & Compensation frequency band & 1 \\
\hline$T_{L}$ & Compensation rate & 3 \\
\hline$\tau_{p}$ & Human processing time delay & 0.04 \\
\hline$K_{r}$ & Steering column stiffness (internal model) & 1 \\
\hline$K_{t}$ & Steering wheel holding stiffness & 12 \\
\hline$T_{N}$ & Neuromuscular time constant & 0.1 \\
\hline
\end{tabular}

designed according to a multi-objective $H_{2} / H_{\infty}$ strategy. Criteria and constraints are defined to deal with compromises between trajectory tracking, driver comfort, sharing level and robustness properties of the feedback control law. Finally, simulation results and robustness are analysed in Section IV, followed by some conclusions and perspectives in Section V.

\section{DRIVER-VEHICLE-ROAD MODEL DESCRIPTION}

\section{A. Driver model}

It has been shown that for haptic shared control, the use of a driver model improves the human-machine interactions [15]. It makes it possible to design a co-pilot more aware of the human behaviour and will, and reciprocally. In this paper such a driver model will be used to design the feedback compensatory controller.

The driver model used here was developed in [8] and [9]. It is shown in Fig. 1 of [9] which distinguishes anticipatory and compensatory parts of this model. This cybernetic model is based on the hypothesis that the driver uses visual perception to get information about the upcoming road curvature. It is commonly admitted that the driver uses two distinct points to steer the vehicle [16]. A near point, located at a short distance in front of the vehicle and a far point which is used to know the road curvature ahead. This point is placed on the tangent point between the driver's gaze direction and the edge of the lane. Parameters used for the driver model are described in Table I. The driver model can be written as:

$$
\left[\begin{array}{c}
x_{1 d} \\
x_{2 d} \\
\dot{\Gamma}_{d}
\end{array}\right]=A_{d}\left[\begin{array}{c}
x_{1 d} \\
x_{2 d} \\
\Gamma_{d}
\end{array}\right]+B_{d}\left[\begin{array}{c}
\theta_{\text {far }} \\
\theta_{\text {near }} \\
\delta_{d} \\
\Gamma_{s}
\end{array}\right]
$$

Where $x_{1 d}$ and $x_{2 d}$ are driver's state components, $\Gamma_{d}$ is the torque applied by the driver, $\theta_{\text {far }} \approx D_{\text {far }} \rho_{\text {ref }}$ is the angle to the tangent point, $\rho_{\text {ref }}$ is the road curvature, $D_{\text {far }}$ is described in Table II, $\theta_{\text {near }}=\psi_{L}+y_{L} / l_{s}$ is the angle to the near point, $\psi_{L}$ is the heading error angle, $y_{L}$ is the lateral error at the lookahead distance $l_{s}$ which can be approximated as $y_{C G}+l_{s} \psi_{L}$ where $y_{C G}$ is the lateral error at the vehicle's center of gravity, $\delta_{d}$ is the steering wheel angle and $\Gamma_{s}$ is the selfaligning torque which can be found as :

$$
\Gamma_{s}=-\frac{2 K_{m} C_{f} \eta_{t}}{R_{s}}\left(\beta+\frac{l_{f}}{V_{x}} r-\frac{1}{R_{s}} \delta_{d}\right)
$$

$A_{d}$ and $B_{d}$ are matrices described in [8] using parameters shown in Table I.

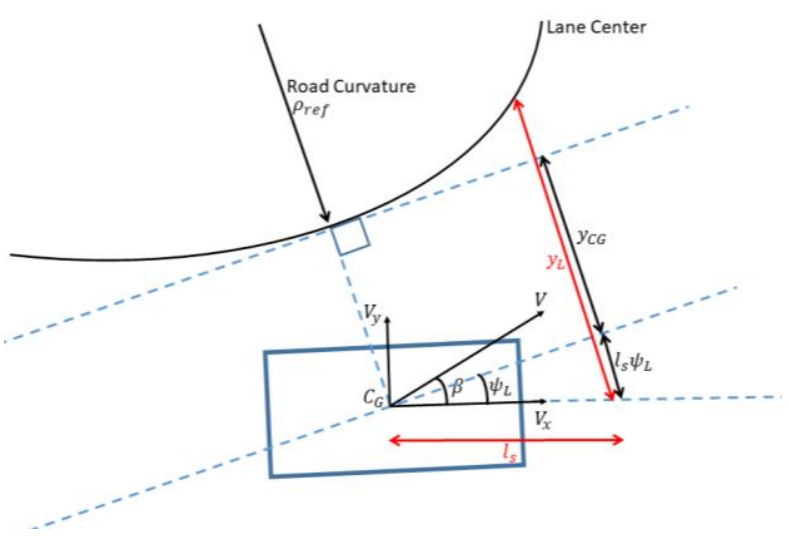

Figure 1. Vehicle position on the road

TABLE II. VEHICLE MODEL PARAMETERS

\begin{tabular}{|c|c|c|}
\hline$l_{f}$ & $\begin{array}{c}\text { Distance from gravity center to } \\
\text { front axle }\end{array}$ & $1.127 \mathrm{~m}$ \\
\hline$l_{r}$ & $\begin{array}{c}\text { Distance from gravity center to } \\
\text { rear axle }\end{array}$ & $1.485 \mathrm{~m}$ \\
\hline$m$ & Total mass & $1476 \mathrm{Kg}$ \\
\hline$J$ & Vehicle yaw moment of inertia & $1810 \mathrm{Kg} \cdot \mathrm{m}^{2}$ \\
\hline$C_{f 0}$ & Front cornering stiffness & $65000 \mathrm{~N} / \mathrm{rad}$ \\
\hline$C_{r 0}$ & Rear cornering stiffness & $57000 \mathrm{~N} / \mathrm{rad}$ \\
\hline$\eta_{t}$ & Tire length contact & $0.185 \mathrm{~m}$ \\
\hline$\mu$ & Adhesion & 0.8 \\
\hline$K_{m}$ & Manual steering column gain & 0.13 \\
\hline$R_{S}$ & Steering gear ratio & 16 \\
\hline$B_{S}$ & $\begin{array}{c}\text { Steering system damping } \\
\text { coefficient }\end{array}$ & 5.7 \\
\hline$I_{S}$ & Inertial moment of steering & $0.05 \mathrm{Kg} \cdot \mathrm{m}^{2}$ \\
\hline$l_{s}$ & system & $5 \mathrm{~m}$ \\
\hline$V_{x}$ & Look-ahead distance & $18 \mathrm{~m} . \mathrm{s}^{-1}$ \\
\hline$D_{f a r}$ & Distance to the tangent point & $15 \mathrm{~m}$ \\
\hline
\end{tabular}

\section{B. Vehicle-Road model}

A vehicle-road model will be necessary to tune control laws used in the feedforward and the feedback parts, but also to simulate the virtual vehicle. The longitudinal speed of the vehicle $V_{x}$ is assumed constant. This vehicle-road model describes vehicle dynamics, including vehicle's lateral dynamics using the classic bicycle model, steering column, and position of the vehicle on the road. Keeping notation introduced in [14], it can be described as follows:

$$
\dot{x}_{v r}=A_{v r} x_{v r}+B_{1 v r}\left(\Gamma_{a}+\Gamma_{d}\right)+B_{2 v r} \rho_{r e f}+B_{3 v r} F_{w}
$$

with $x_{v r}=\left[\begin{array}{lllll}\beta & r & \psi_{L} & y_{L} & \delta_{d} \\ \delta_{d}\end{array}\right]^{T}$, in which $\beta$ is the side split angle, $r$ the yaw rate. Input signals are $\rho_{\text {ref }}$ the road curvature, $F_{w}$ the side wind resultant applied on the vehicle's center of gravity, $\Gamma_{a}$ the assistance torque applied on the steering wheel and $\Gamma_{d}$ the driver torque. Matrices $A_{v r}, B_{1 v r}, B_{2 v r}$ and $B_{3 v r}$ are described in [3], [10] using vehicle model parameters depicted in Table II and Fig. 1. $l_{w}$ is the distance between the vehicle's center of gravity and another point on which the wind force is applied. 


\section{Driver-Vehicle-Road global model}

Finally, the model used to describe the whole system can be found using both driver and vehicle-road models. This model is adjusted, as driver and vehicle-road model, to the longitudinal speed $V_{x}$. The global state space model, which is an LTI system, can be written as follows:

with

$$
\left\{\begin{array}{c}
\dot{x}_{d v r}=A_{d v r} x_{d v r}+B_{d v r} U_{d v r} \\
y_{d v r}=C_{d v r} x_{d v r}
\end{array}\right.
$$

$$
\begin{aligned}
& x_{d v r}=\left[\begin{array}{llllll}
\beta r & \psi_{L} & y_{L} \delta_{d} \dot{\delta}_{d} & x_{1 d} & x_{2 d} & \Gamma_{d}
\end{array}\right]^{T}, \\
& U_{d v r}=\left[\begin{array}{lll}
\Gamma_{a} & \rho_{\text {ref }} & F_{w}
\end{array}\right]^{T}, y_{d v r}=\left[\begin{array}{llll}
\beta r & \psi_{L} & y_{L} \delta_{d} \dot{\delta}_{d}
\end{array}\right]^{T},
\end{aligned}
$$

Notice that the two input signals $\theta_{\text {near }}$ and $\theta_{\text {far }}$ have to be expressed in terms of their relationship with the vehicle-road signals (see II.A.). Matrices $A_{d v r}, B_{d v r}$ and $C_{d v r}$ are described in [3], [10].

\section{ARCHITECTURE}

Fundamentally, to carry out the steering task, the system needs to execute two different roles: an anticipation to predict changes in road profile and a correction to adjust vehicle position to the lane center (or any other chosen trajectory, for example while operating a lane change). In the control strategy developed in this article, these roles are explicitly separated in two parts, to make the system more adjustable with a possibly different level of sharing for each part. This separation allows to control the balance between the compensatory and the anticipatory parts, which brings the system behaviour closer to how humans drive. Indeed, in [17], it is shown that drivers can adapt their steering strategy depending on visual cues received by modifying the balance between these two parts.

Fig. 2 displays the global architecture proposed here. The anticipatory e-assistant is encapsulated in the green dotted rectangle while the compensatory one is in the red dotted rectangle. The yellow square $\mathrm{T} 1$ materialises the

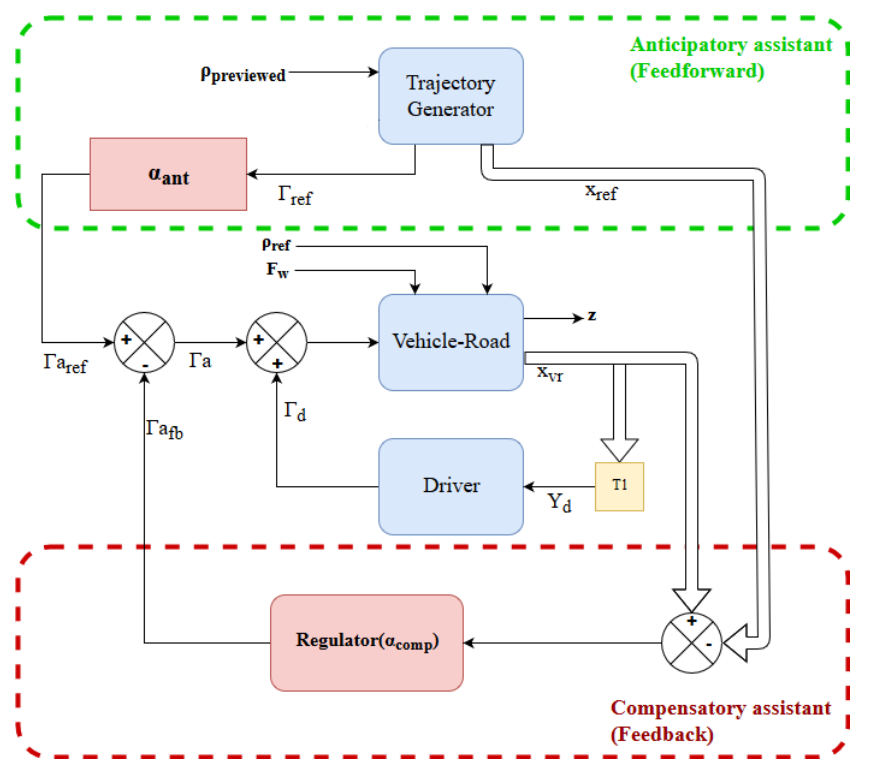

Figure 2. Shared control strategy transformation applied to the state of the vehicle-road model to get the driver model inputs $Y_{d}=\left[\begin{array}{llll}\theta_{\text {far }} & \theta_{\text {near }} & \delta_{d} & \Gamma_{s}\end{array}\right]^{T}$, equations used are described in Section II-A. Red boxes show how the levels of sharing may be tuned, respectively in the anticipatory and compensatory parts through the gains $\alpha_{\text {ant }}$ and $\alpha_{\text {comp }}$. These parameters can be chosen to be equal, as it is implicitly the case in [3], or different, to emphasize the compensatory or the anticipatory behaviour of the assistance.

The anticipation part, described in Section IV.A, is based on a trajectory generator simulating a virtual autonomous vehicle. The trajectory of the latter (vehicle-road states and related steering torque) is then considered as the reference trajectory for the real car. On the other side, the compensatory

part, described in Section IV.B, consists of a $H_{2} / H_{\infty}$ static output feedback that controls the steering and level of sharing against disturbances and uncertainties. As in [3] to make the comparison fair, it is assumed here that all states of the vehicle-road system are available. On the other hand, the states of the pilot model are not supposed to be known, contrary to what is supposed in [14], [3]. However, the design method proposed here adapts very directly to more restrictive conditions as to the unavailability of some sensors.

\section{DESIGN Methodology}

\section{A. Anticipatory algorithm}

The objective of this part of the system is to provide a reference trajectory generator for the car. This has to be done by knowing the road geometry, further on a short time ahead. More precisely, the assumption is that the reference trajectory is known from the road curvature only, while the control reference and the related vehicle-road reference state (which constitute the whole trajectory reference) have to be determined. Different ways may be considered to meet this goal, among which the one proposed in [18] which looks for gains linking a constant curvature to the main vehicle states (steering angle, the heading angle, the yaw rate...).

The reference trajectory generator proposed here makes use of a more general principle, allowing to cope with the current study, whose control input is the steering torque rather than the steering angle. Inspired by the works on dynamic system inversion, the solution proceeds by using both the feedback principle and simulation. Precisely, the trajectory generator proposed now relies on online simulation of a virtual autonomous vehicle that uses the vehicle road model proposed in Section II.B. By using the simulation of a virtual vehicle through using a model consistent with the real vehicle, the generated trajectory is assured to be dynamically feasible for the real vehicle [13], [19]. This virtual vehicle is forced to follow the (possibly virtual) given curvature path by simulating as feedback the $\mathrm{H}_{2}$-preview controller designed in [14]. This is done according to Fig. 3. Let's note that the robustness issues within the closed-loop system used in the inverse simulation are irrelevant because there are no uncertainties or external disturbances (except numerical ones). 


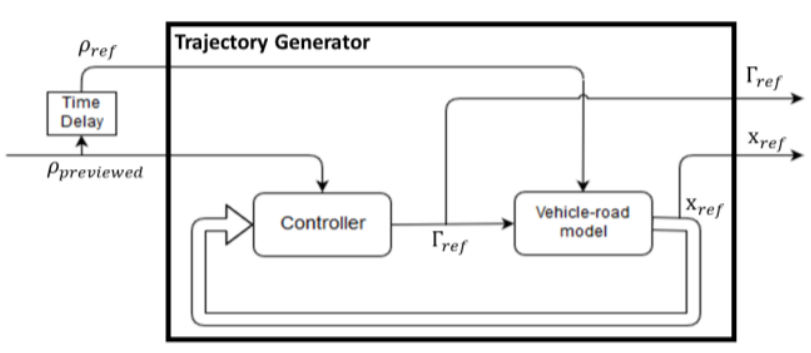

Figure 3. reference trajectory generation through inverse simulation

As shown in Fig. 2, the input of the anticipatory part is $\rho_{\text {previewed }}$, it represents the road curvature reference previewed at the time $t+T_{\text {horizon }}: \rho_{\text {ref }}(t)=\rho_{\text {previewed }}(t+$ $\left.T_{\text {horizon }}\right)$. With $t$ the current time and $T_{\text {horizon }}$ is the anticipation time. Outputs are the state of the virtual vehicle-

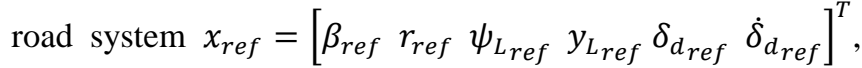
and the related torque leading to it, $\Gamma_{r e f}$ which is then scheduled by the level of sharing of the anticipatory part: $\Gamma a_{\text {ref }}=\alpha_{\text {ant }} \Gamma_{\text {ref }}$.

The virtual vehicle is controlled through a $\mathrm{H}_{2}$-Preview method described in [14] which is an excellent candidate for this part as it manages preview on the road curvature, but other methods can be used. Another strategy that might have been used is an MPC algorithm but the $H_{2}$-Preview solution has the benefit to supply an analytical solution which mean that the calculation cost will be low compare to when using an MPC.

\section{B. Compensatory algorithm}

In [3], the compensatory part of the $H_{2}$-Preview control used is carried out by a state-feedback term using the drivervehicle-road system state. An observer then estimates driver state values, that can't be measured. In the lateral assistance proposed here, the compensatory part is based on a static output feedback synthesis. One important advantage is flexibility with regards to the measures available, which is essential for practical (implementation) reasons. This has a price: the control synthesis problem is then non-convex [20]. For comparison reasons, the output considered for feedback are chosen as close as possible to the configuration used in [3]. The main difference is that it becomes useless to estimate the driver model states.

Fig. 2 highlights the compensatory and anticipatory parts work together. The output feedback from the compensatory part is applied to the deviation between the real vehicle-road system state and the virtual one:

$$
\begin{gathered}
\Gamma_{a_{f b}}=K \times Y_{\text {diff }}=k_{\beta} \beta_{\text {diff }}+k_{r} r_{\text {diff }}+k_{\psi_{L}} \psi_{L \text { diff }}+ \\
k_{y_{L}} y_{L_{\text {diff }}}+k_{\delta_{d}} \delta_{d_{\text {diff }}}+k_{\delta_{d}} \dot{\delta}_{d_{\text {diff }}}
\end{gathered}
$$

with $Y_{\text {diff }}=x_{v r}-x_{\text {ref }}$.

To design this static output feedback efficiently, a $H_{2} / H_{\infty}$ multi-objective control synthesis was used. The $\mathrm{H}_{2}$ criterion is employed, as in [10], to define the control performance, including the sharing quality indicators. The $H_{\infty}$ norm is used to guarantee some unstructured robustness, by bounding

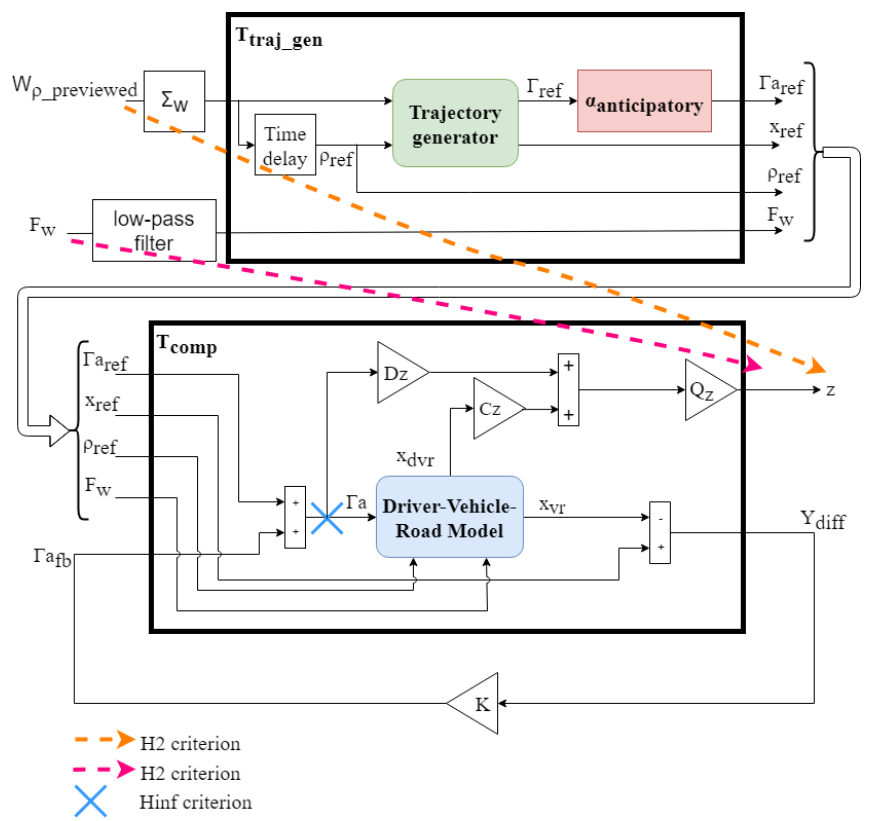

Figure 4. Model used for multi-criteria optimisation

sensitivity functions ( $c f$. classical circle criterion and gainphase margin).

Let us consider the designed model shown in Fig. 4, defined through the following relationships:

$$
\begin{aligned}
& T_{z w}(s)=T_{\text {comp }}(s) T_{\text {traj_gen }}(s) \\
& w=\left[\begin{array}{ll}
\rho_{\text {previewed }} & F_{w}
\end{array}\right]^{T}, \\
& w_{\text {traj }}=\left[\begin{array}{llll}
\Gamma a_{\text {ref }} & x_{\text {ref }} & \rho_{\text {ref }} & F_{w}
\end{array}\right]^{T}, \\
& z=Q_{z}\left[\begin{array}{llllll}
\psi_{L} & y_{C G} & a & \left(\Gamma_{a}-\alpha_{c o m p} \Gamma_{d}\right) & \Gamma_{d} & \Gamma_{a}
\end{array}\right]^{T} . \\
& w_{\text {traj }}=T_{\text {traj_gen }} w, \quad z=T_{\text {comp }} w_{\text {traj }},
\end{aligned}
$$

$T_{\text {comp }}$ can be decomposed in column as:

$$
T_{\text {comp }}=\left(\begin{array}{llll}
T_{\Gamma a_{\text {ref }}} & T_{x_{\text {ref }}} & T_{\rho_{\text {ref }}} & T_{F_{w}}
\end{array}\right)
$$

with

$$
\begin{gathered}
T_{\Gamma a_{r e f}}=Q_{z}\left[C_{z}-\Lambda_{1} C_{d v r}\right] \Lambda_{2} B_{\Gamma_{a}}, \\
T_{x_{r e f}}=Q_{z} \Lambda_{1}, \\
T_{\rho_{r e f}}=Q_{z}\left[C_{z}-\Lambda_{1} C_{d v r}\right] \Lambda_{2} B_{\rho_{r e f}}, \\
T_{F_{w}}=Q_{z}\left[C_{z}-\Lambda_{1} C_{d v r}\right] \Lambda_{2} B_{F_{w}} \\
\Lambda_{1}=\left(C_{z} \Lambda_{2} B_{\Gamma_{a}}+D_{z}\right)\left(I+K C_{d v r} \Lambda_{2} B_{\Gamma_{a}}\right)^{-1} K, \\
\Lambda_{2}=\left(p I-A_{d v r}\right)^{-1},
\end{gathered}
$$

$\rho$, the road curvature, and $F_{w}$, the wind force, are modelled by means of two generator models, $\Sigma_{\rho}$ for $\rho$, proposed in [3], and $\Sigma_{F_{w}}$ for $F_{w}$, a filter amplifying the low frequencies and attenuating the high frequencies.

The $H_{2}$ criterion is described using the weighting matrix $Q_{z}$ defined as:

$$
Q_{z}=\left[\begin{array}{cccccc}
c_{1} & 0 & 0 & 0 & 0 & 0 \\
0 & c_{2} & 0 & 0 & 0 & 0 \\
0 & 0 & c_{3} & 0 & 0 & 0 \\
0 & 0 & 0 & c_{4} & 0 & 0 \\
0 & 0 & 0 & 0 & c_{5} & c_{d a} \\
0 & 0 & 0 & 0 & 0 & 1
\end{array}\right]
$$


This criterion involves elements to manage performance for the lane following task like heading error angle $\psi_{L}$, lateral error at the vehicle's center of gravity $y_{C G}$ and lateral acceleration $a$ which is also a comfort criterion for the driver. Other parameters allow to control the assistance torque adjusting the level of sharing to the wanted values: $\alpha_{\text {comp }}=$ $\Gamma_{a} / \Gamma_{d}$. Parameter $c_{d a}$ enables to prevent assistance torque to be opposed to driver torque what would be uncomfortable for the driver [3], [10].

The $H_{\infty}$ criterion is defined using the associated input sensitivity function denoted $S_{\text {input }}$. Input gain-phase (or module) margin $M m_{\text {input }}$ is then defined as:

$$
M m_{\text {input }}=\frac{1}{\max _{\omega}\left(\left|S_{\text {input }}(j \omega)\right|\right)}=\frac{1}{\left\|S_{\text {input }}\right\|_{\infty}} .
$$

Problem P1: $\mathrm{H}_{2} / \mathrm{H}_{\infty}$ static output feedback design

The $H_{2} / H_{\infty}$ static output feedback design is defined as;

find $K$ such that:

- the driver-vehicle-road system is internally stabilized,

- is the solution of

$$
\begin{aligned}
& \min _{K}\left(\left\|T_{Z w}\right\|_{2}\right) \\
& \text { under the constraint }\left\|S_{\text {input }}\right\|_{\infty}<S_{\text {max }},
\end{aligned}
$$

with $S_{\max }$ a constant defined a priori.

To design a static output feedback is not a trivial problem [20]. It is proposed here to use recent tools such as Systune, available in Matlab ${ }^{\circledR}[21]$, that permits to solve some nonconvex control problems by using non-smooth optimisation algorithms. Initial values are randomized by means of the "RandomStart" option of Systune.

\section{Simulation RESULTS}

\section{A. Tuning parameters}

To validate the assistance architecture and the associated design methodology, we carried out simulations under Matlab/Simulink ${ }^{\circledR}$, with the following tuning. For the anticipatory part, the virtual model is based on (1), without taking into consideration the disturbance input associated to the wind $F_{w}$ (ideal trajectory). The same vehicle-road model (1) is used to simulate the "real" vehicle. And in the same manner, the driver model is both used in the design problem P1 and to simulate the "real" driver.

Concerning the compensatory part, associated to the design problem $\mathrm{P} 1$, the following criteria are used. Matrix $Q_{z}$ (4) is tuned as $c_{1}=200, c_{2}=20, c_{3}=3, c_{4}=5, c_{5}=1$ and $c_{d a}=-10$. The desired input modulo margin $M m_{\text {input }}$ is 0.5 . Finally, the two level of sharing $\alpha_{\text {ant }}$ and $\alpha_{\text {comp }}$ are tuned both to obtain a level of sharing of $50 \%$. The constant longitudinal speed of the real and virtual vehicle is set at $V_{x}=$ $18 \mathrm{~m} / \mathrm{s}$. Notice that these tuning values come from the previous results on the shared control assistance based on the $\mathrm{H}_{2}$-preview [3]. As said previously, our first objective here is to validate that this architecture, more modular, leads to the same satisfactory behaviour than the $\mathrm{H}_{2}$ controller in terms of time performance and comfort (sharing quality in particular), but also robustness (intrinsic LQR input gain-phase margin).
The track used for this simulation was saved from Satory test track in Versailles showed in Fig. 5.

\section{B. Time performances and sharing indicators}

Indicators used to evaluate system's performance in terms of sharing quality are introduced in [3] and [14]:

- Consistency ratio, $T_{c o}$, defined as the duration during which assistance torque $\Gamma_{a}$ is in the same direction as the driver torque $\Gamma_{d}$ divided by simulation's duration.

- Resistance ratio, $T_{\text {res }}$, defined as the duration during which assistance torque $\Gamma_{a}$ and driver torque $\Gamma_{d}$ are in opposite direction and assistance torque is inferior or equal to the driver torque divided by simulation's duration

- Contradiction ratio, $T_{\text {cont }}$, defined as the duration during which assistance torque $\Gamma_{a}$ and driver torque $\Gamma_{d}$ are in opposite direction and assistance torque is higher than the driver torque divided by simulation's duration.

- Sharing level is defined as effort produced by the assistance divide by effort produced by the driver

$$
P_{m}=\frac{E_{a}}{E_{d}}=\frac{\int_{0}^{\infty} \Gamma_{a}^{2}(t) d t}{\int_{0}^{\infty} \Gamma_{d}^{2}(t) d t}
$$

- Coherence level that is the cosine value of the angle between the assistance torque and the driver torque

$$
P_{c}=\cos \left(\overrightarrow{\Gamma_{a}}, \overrightarrow{\Gamma_{d}}\right)=\frac{\int_{0}^{\infty} \Gamma_{a}(t) \times \Gamma_{d}(t) d t}{\sqrt{\int_{0}^{\infty} \Gamma_{a}^{2}(t) d t \times \int_{0}^{\infty} \Gamma_{d}^{2}(t) d t}}
$$

Three other indicators are used to evaluate lane following performances: maximum, average and standard deviation values of lateral error during the simulation. Results found here are compared with results found during the simulation of the driver alone and the simulation of the driver assisted by system developed in [3]. Reminds that this previous assistance is a shared steering control developed using also the drivervehicle-road model, but that the driver action is used in the $\mathrm{H}_{2}$ /LQ state feedback via an observer.

\section{Results}

Table III displays results of simulations done in the three following cases. In the first column, the driver is driving without assistance. In column (a), the driver is assisted by the system developed in [3]. This system is going to be called assistance (a) in the following. For last column, the driver is assisted by the system developed in this paper that is going to be called assistance (b). Fig. 6 shows the different torques applied to the system for the two assistances (a) and (b). The green line corresponds to the torque applied by the driver, the red one to the torque applied by the assistance and the black one is the total torque which is the sum of the driver and the assistance ones.

First, note on Fig. 6 around 30 seconds, the very high torque. See also the importance of maximal error in Table III, especially without assistance. This is due to the (too) rapid passage of a tight curve, which has the merit of being discriminatory if not realistic. Table III shows that both assistance (a) and (b) improve lane following performance when compared with no assistance, and in similar proportions. 


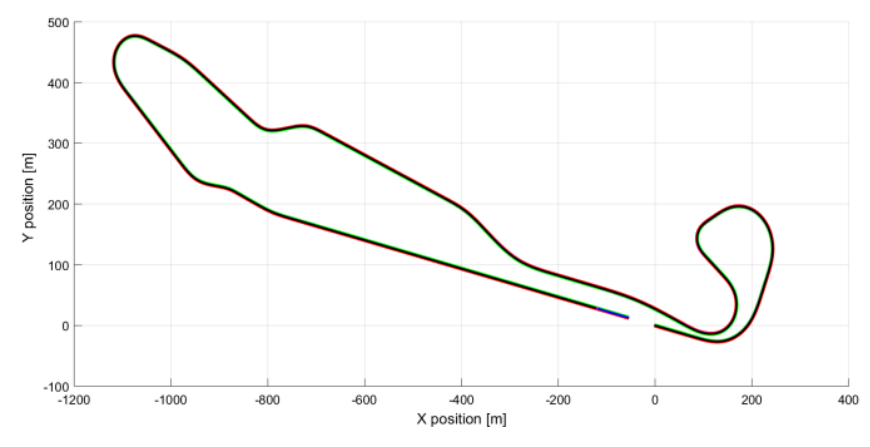

Figure 5. Satory test track

TABLE III. SimULATION RESULTS

\begin{tabular}{|c|c|c|c|}
\hline & Driver & (a) & (b) \\
\hline max(lateral_error)(m) & 3,02 & 1,37 & 1,37 \\
\hline average(lateral_error)(m) & 0,31 & 0,24 & 0,32 \\
\hline Std(lateral_error)(m) & 0,47 & 0,32 & 0,41 \\
\hline$T_{\text {co }}$ & $/$ & 0,47 & 0,70 \\
\hline$T_{\text {res }}$ & $/$ & 0,43 & 0,14 \\
\hline$T_{\text {cont }}$ & $/$ & 0,10 & 0,29 \\
\hline$P_{m}$ & $/$ & 0,25 & 1.02 \\
\hline$P_{c}$ & $/$ & 0,82 & 0,90 \\
\hline
\end{tabular}

Results found are close for both assistances in terms of lane following criteria.

At the same time, the sharing performance are good for the two assistances with a consistency ratio higher than the resistance and the contradiction ratios. The coherence level, $P_{c}$ which is very close to 1 also indicates that the driver and the assistance are mostly acting in accordance with each other. Otherwise, the consistent ratio is higher using assistance (b) than assistance (a).

Finally, Fig 6. shows that the shape of the assistance torque is similar in both cases. The assistance torque is a little less important for assistance (a), but the general properties are retained. It seems that the assistance developed in this article has a coherent action compared with this previous steering control system.

\section{Robustness analysis}

Values found when optimizing the optimization problem P1 defined in Section IV.B. are : $k_{\beta}=47.72, k_{r}=3.99$, $k_{\psi_{L}}=4.45, k_{y_{L}}=0.27, k_{\delta_{d}}=-1.06$ and $k_{\dot{\delta}_{d}}=-0.82$. It results in an input gain-phase margin of 0.63 . This margin is important, that means a good overall robustness.

\section{E. Discussion and perspectives}

Section V shows that results found with the system developed in this article, although obtained in a different way, are mainly close to the ones found with assistance (a). This result is particularly interesting because the new formulation has a strong ability to evolve, whether it is the structure of the assistance regulator (a state observer is not required), or the objectives and constraints taken into account. Moreover, the consistency ratio and the coherence level are high which means that sharing performance is good. (a)

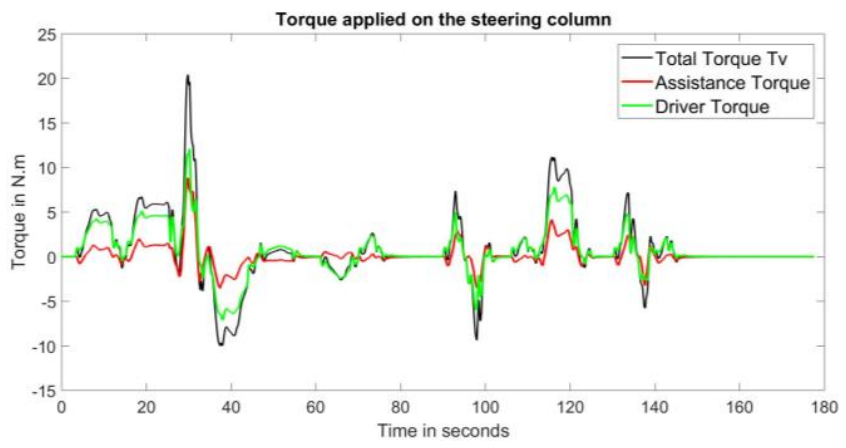

(b)

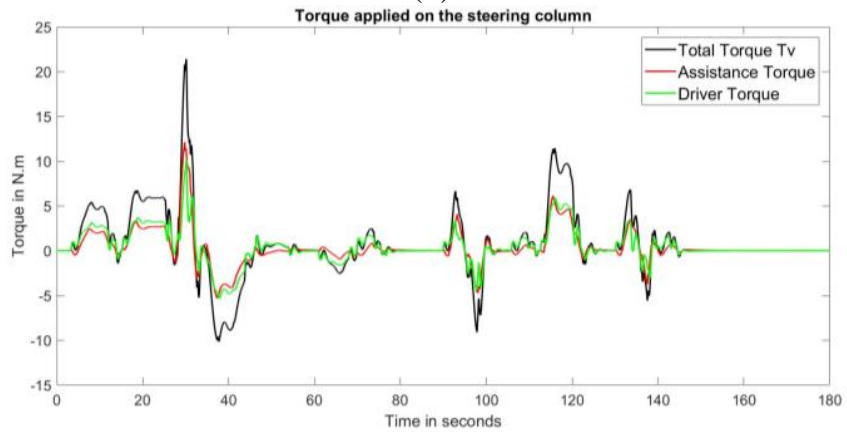

Figure 6. Torque applied on the steering column

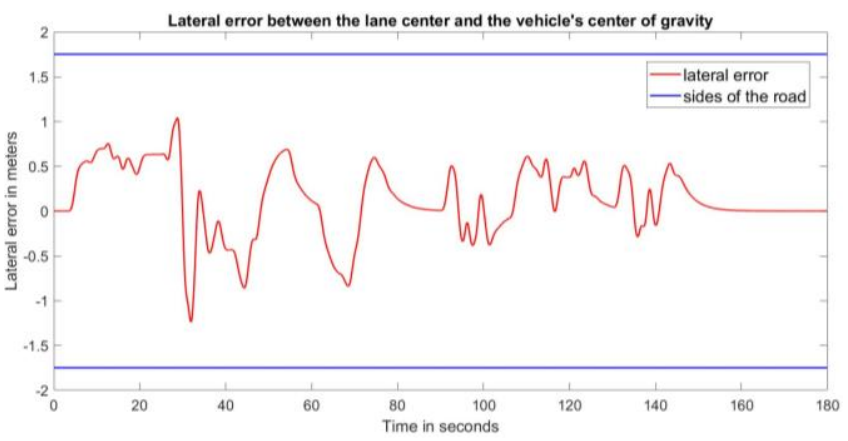

Figure 7. Lateral error during the simulation

However, the system described here can only deal with constant longitudinal speed as it was synthesized at a fixed longitudinal speed, $V_{x}$. It can be improved by making it able to be operational while modifying the longitudinal speed for example by using gain scheduling [22] or an LPV approach [23].

Another adaptation that can be easily done with this architecture is allowed by the use of an output feedback in the compensatory part. It allows to only use needed and/or technologically available values for the feedback instead of using the entire system's state values. This characteristic is interesting since all values of the driver-vehicle-road system can't be measured, besides it allows to develop a functional system without using observer to approximate missing values. It calls to make a study on which signals are intrinsically necessary to consider in the feedback loop.

This system architecture in two parts gives also many perspectives. First, the use of separated sharing level for both parts can give the opportunity to investigate the use of different assistance behaviour to have an assistance that acts 
TABLE IV. SIMULATION RESULTS

\begin{tabular}{|c|c|c|c|}
\hline & $20 \%$ & $50 \%$ & $70 \%$ \\
\hline max(lateral_error)(m) & 2,15 & 1,39 & 1,09 \\
\hline average(lateral_error)(m) & 0,28 & 0,32 & 0,34 \\
\hline Std(lateral_error)(m) & 0,40 & 0,41 & 0,43 \\
\hline$T_{\text {co }}$ & 0,68 & 0,68 & 0,60 \\
\hline$T_{\text {res }}$ & 0,29 & 0,28 & 0,34 \\
\hline$T_{\text {cont }}$ & 0,03 & 0,04 & 0,06 \\
\hline$P_{m}$ & 0,06 & 0,81 & 3,37 \\
\hline$P_{c}$ & 0,98 & 0,94 & 0,87 \\
\hline
\end{tabular}

more like an anticipatory system or a compensatory system. For example, it can adapt according to the visual context of the driving situation as a human would, according to [17]. It can lead to an assistance behavior closer to the human one.

At last, the anticipatory part, which rests on a trajectory generator algorithm could be adapted to be used in situations that require to define another trajectory to follow rather than the lane center. For example, in the case of a lane change, the reference trajectory can be adapted to meet this expectation.

\section{F. Perspectives as a mean to do transition}

In this section, the control strategy developed above was tested using the same tuning parameters as in the previous simulation but with a different $H_{2}$ criterion and different values of level of sharing defined as $\alpha=\alpha_{\text {ant }}=\alpha_{\text {comp }}$. A first simulation was done with $\alpha=20 \%$, that is to say that the assistance should supply $20 \%$ of the torque needed to steer the vehicle. Two other simulations were carried out with $\alpha=$ $50 \%$ and $\alpha=70 \%$.

The vector $z$ was modified to allow the level of sharing to go from 0 to $100 \%$. Indeed, with the previous criterion designed in [10] and used in Section IV-B, $\alpha_{\text {comp }}=\frac{\alpha}{1-\alpha}$ was found using these two equations :

$$
\alpha=\frac{\Gamma_{a}}{\Gamma_{a}+\Gamma_{d}}, \quad \Gamma_{a}-\alpha_{c o m p} \Gamma_{d}=0
$$

This formula doesn't allow $\alpha$ to reach $100 \%$. Then, in this section, the vector $\mathrm{z}$ was taken as:

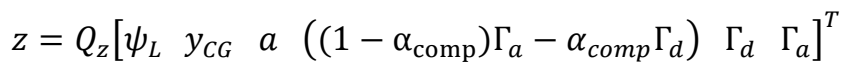

Table IV displays indicators for the three different level of sharing values considered. It shows that the consistency ratio and the coherence level decrease slightly as the level of sharing increase but these values are still showing that the assistance acts most of the time in coherence with the driver. Moreover, the maximal lateral error decline as the assistance supply a higher contribution to the steering torque and the average and standard deviation values remained low. Finally, the sharing level $P_{m}$ raises with $\alpha$ which confirms that the assistance provides a greater effort when the level of sharing becomes higher.

To conclude, this part shows that the architecture developed in this article can be used at different fixed level of sharing values. Then, making it able to work when this value is moving can be very interesting in order to investigate the use of this system to do transition between autonomous and manual driving.

\section{CONCLUSION}

This paper has proposed an innovative shared control strategy for car lateral control. The control co-driving the steering is based on an architecture separated in two distinct parts. The first proceeds by anticipation and is mainly concerned by the road geometry, while the second has to react against unforeseen effects. Both take into account the driver's expectations and the vehicle dynamics, at least indirectly through the use of ad hoc models. This separation allows fixing distinct levels for the anticipatory and compensatory parts in sharing activity with the driver.

The anticipatory part proposed is based on an algorithm acting as an autonomous pilot. In order to generate a reference trajectory, it simulates in parallel to the progression of the real car, a virtual one controlled thanks to an existing $\mathrm{H}_{2}$-Preview controller. The compensatory part is synthetized from a driver-vehicle-road model as a mixed $H_{2} / H_{\infty}$ control problem, taking into account not only lateral control performance, but also several indicators of the quality of the assistance-driver interaction. This is made possible using a driver cybernetic model previously developed. Technically, the multi-objective optimization problem considered for the compensatory part is non-convex and non-smooth. Using subgradients as proposed in Apkarian, proved to be efficient for such a problem, with no main difficulty due to only local convergence property. The steering assistance proposed was compared to the $\mathrm{H}_{2}$ Preview shared control strategy developed in [3] and [10]. It results in similar performance, with efficient lane following and good sharing properties, this although no observer were needed and the fact that the new strategy presents better possibilities for evolution.

Finally, this strategy opens up many opportunities. It can be adapted to practical situations (information available through sensors, uncertainties, etc.) while taking into account a rich panel of sharing's quality indicators. It will also permit to study the incidence on the driver perception of a different sharing level at anticipatory and compensatory levels. This is totally new. Finally, it will be useful in the context of autonomous vehicles development, to manage smooth transitions between autonomous and manual driving, this in more general situations such as lane change.

\section{ACKNOWLEDGMENTS}

This work was supported by AutoConduct research program funded by the French ANR “Agence Nationale de la Recherche” (grant ANR-16-CE22-0007-05).

\section{REFERENCES}

[1] D. A. Abbink et al., «A Topology of Shared Control Systems-Finding Common Ground in Diversity », IEEE Trans. Hum.-Mach. Syst., vol. 48, no 5, p. 509-525, oct. 2018.

[2] P. Griffiths et R. B. Gillespie, « Shared control between human and machine: haptic display of automation during manual control of vehicle heading », in $12 \mathrm{th}$ 
International Symposium on Haptic Interfaces for Virtual Environment and Teleoperator Systems, 2004. HAPTICS '04. Proceedings, 2004, p. 358-366.

[3] L. Saleh, P. Chevrel, F. Claveau, J. F. Lafay, et F. Mars, «Shared Steering Control Between a Driver and an Automation: Stability in the Presence of Driver Behavior Uncertainty », IEEE Trans. Intell. Transp. Syst., vol. 14, p. 974-983, juin 2013.

[4] M. A. Benloucif, C. Sentouh, J. Floris, P. Simon, et J.C. Popieul, «Online adaptation of the Level of Haptic Authority in a lane keeping system considering the driver's state », Transp. Res. Part F Traffic Psychol. Behav., sept. 2017.

[5] D. A. Abbink, M. Mulder, et E. R. Boer, « Haptic shared control: smoothly shifting control authority? », Cogn. Technol. Work, vol. 14, no 1, p. 19-28, mars 2012.

[6] C. Sentouh, B. Soualmi, J. C. Popieul, et S. Debernard, " Cooperative Steering Assist Control System », in 2013 IEEE International Conference on Systems, Man, and Cybernetics, 2013, p. 941-946.

[7] M. Flad, L. Fröhlich, et S. Hohmann, « Cooperative Shared Control Driver Assistance Systems Based on Motion Primitives and Differential Games », IEEE Trans. Hum.-Mach. Syst., vol. 47, no 5, p. 711-722, oct. 2017.

[8] L. Saleh, P. Chevrel, F. Mars, J.-F. Lafay, et F. Claveau, "Human-like cybernetic driver model for lane keeping », IFAC Proc. Vol., vol. 44, n 1, p. 4368-4373, janv. 2011.

[9] F. Mars et P. Chevrel, « Modelling human control of steering for the design of advanced driver assistance systems », Annu. Rev. Control, vol. 44, p. 292-302, janv. 2017.

[10] L. Saleh, "Contrôle Latéral Partagé d'un Véhicule Automobile », phdthesis, Ecole Centrale de Nantes (ECN), 2012.

[11] N. R. Kapania et J. C. Gerdes, « Design of a feedbackfeedforward steering controller for accurate path tracking and stability at the limits of handling », Veh. Syst. Dyn., vol. 53, no 12, p. 1687-1704, déc. 2015.

[12] R. Attia, J. Daniel, J. P. Lauffenburger, R. Orjuela, et M. Basset, « Reference generation and control strategy for automated vehicle guidance », in 2012 IEEE Intelligent Vehicles Symposium, 2012, p. 389-394.

[13] Y. Kuwata, J. Teo, S. Karaman, G. Fiore, E. Frazzoli, et J. How, « Motion Planning in Complex Environments Using Closed-loop Prediction », in AIAA Guidance, Navigation and Control Conference and Exhibit, 0 vol., American Institute of Aeronautics and Astronautics, 2008.

[14] L. Saleh, P. Chevrel, et J.-F. Lafay, « Generalized H2Preview Control and its Application to Car Lateral Steering », IFAC Proc. Vol., vol. 43, n 2, p. 132-137, janv. 2010.

[15] D. A. Abbink et M. Mulder, « Exploring the Dimensions of Haptic Feedback Support in Manual Control », $J$. Comput. Inf. Sci. Eng., vol. 9, n ${ }^{\circ}$ 1, p. 011006, mars 2009.
[16] D. Salvucci et R. Gray, «A two-point visual control model of steering », Perception, vol. 33, p. 1233-48, févr. 2004.

[17] I. Frissen et F. Mars, « The Effect of Visual Degradation on Anticipatory and Compensatory Steering Control », Q. J. Exp. Psychol., vol. 67, nº 3, p. 499-507, mars 2014.

[18] R. Rajamani, Vehicle Dynamics and Control, New York, NY, USA: Springer, 2012.

[19] F. Claveau, P. Chevrel, et K. Knittel, «A 2DOF gainscheduled controller design methodology for a multimotor web transport system », Control Eng. Pract., vol. 16, no 5, p. 609-622, mai 2008.

[20] M. S. Sadabadi et D. Peaucelle, «From static output feedback to structured robust static output feedback: A survey », Annu. Rev. Control, vol. 42, p. 11-26, janv. 2016.

[21] P. Apkarian, M. N. Dao, et D. Noll, « Parametric Robust Structured Control Design», IEEE Trans. Autom. Control, vol. 60, $\mathrm{n}^{\mathrm{O}}$ 7, p. 1857-1869, juill. 2015.

[22] W. J. Rugh et J. S. Shamma, "Research on gain scheduling », Automatica, vol. 36, $\mathrm{n}^{\circ}$ 10, p. 1401-1425, oct. 2000.

[23] J. Mohammadpour et C. W. Scherer, Control of Linear Parameter Varying Systems with Applications. New York, NY, USA: Springer, 2012. 